\section{$\$$ Research Square}

Preprints are preliminary reports that have not undergone peer review.

They should not be considered conclusive, used to inform clinical practice, or referenced by the media as validated information.

\title{
The Hsp70 Gene family in Solanum Lycopersicum, Genome-wide Identification and Expression Analysis Under Heavy Metals Stresses
}

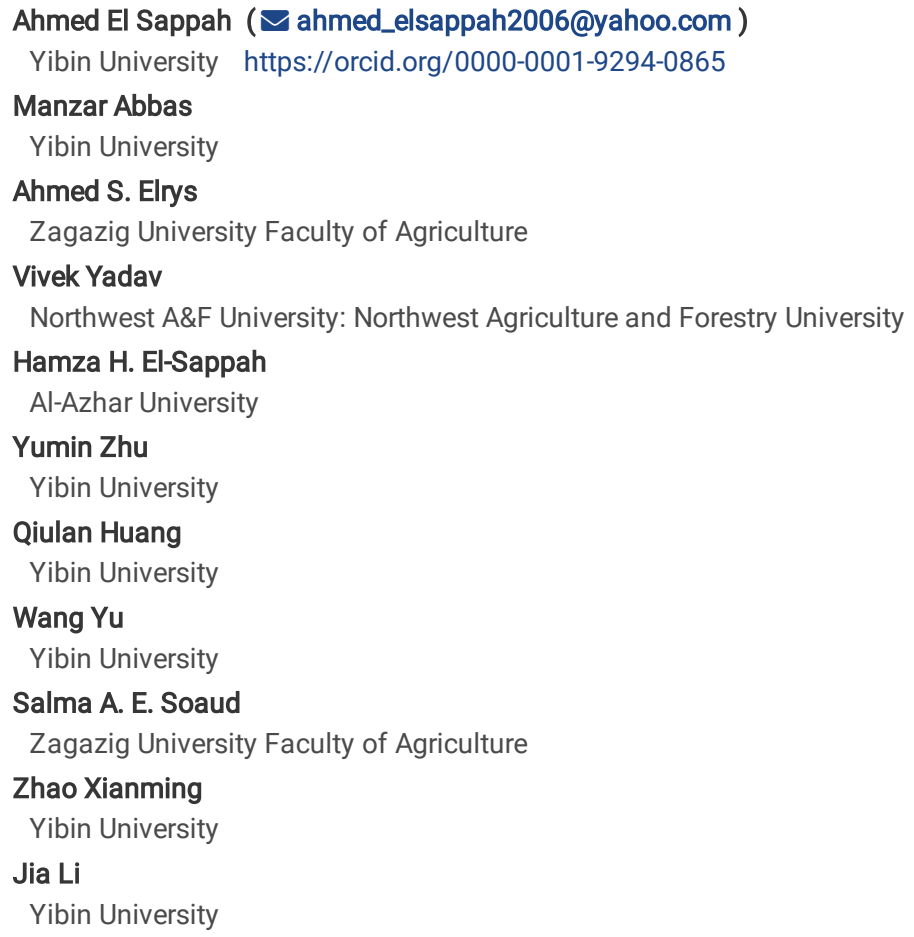




\section{Abstract}

The Heat shock protein-70 (Hsp70) gene family is one of the protective mechanisms; however, it has not been widely studied in tomatoes. Therefore, the current study provides the first report genome-wide analysis of the Hsp70 gene family in tomato (Solanum lycopersicum L.) under five heavy metals (Cd ${ }^{2+}$, $\mathrm{Co}^{2+}, \mathrm{Mn}^{2+}, \mathrm{Zn}^{2+}$ and $\mathrm{Fe}^{2+}$ ) stresses. We identified 23 candidate genes of the $\mathrm{Hsp} 70$ gene family based on the PF00012 domain through bioinformatics studies, including gene structure, distribution, synteny, phylogenetic tree, protein-protein interactions, gene ontology, and previous RNA-seq data analysis followed by qRT-PCR analysis. Based on the phylogenetic analysis, the 23 candidate genes were classified into five subfamilies where the same subfamily contains similar SIHsp70 proteins. Many pairs of SIHsp70 gene duplications have appeared, consisting of tandem and segment duplication. In addition, analysis of previous RNA-seq besides the gene ontology gave us significant evidence about the vital roles of these genes during tomato development and growth. The SIHsp7s showed different responses, which were varied depend on different plant tissues and types of heavy metal. Some of the SIHsp70s were up-regulated after heavy metal exposure, such as $\mathrm{Cd}^{2+} / \mathrm{S} / \mathrm{Hsp} 70-23$ and $\mathrm{Mn}^{2+} / \mathrm{S} / \mathrm{Hsp} 70-8$. Still, down-regulated others such as Fe ${ }^{2+} / \mathrm{SIHsp70-18}$. Finally, our gene expression analysis revealed the significant roles of the Hsp70s, especially, SIHsp70-3, SIHsp70-8, SIHsp70-12, SIHsp70-19, and SIHsp70-23, with the different heavy metals treatments.

\section{Introduction}

Tomato (Solanum lycopersicum L.) is a common and economically essential crop worldwide after potato with 177 million tons production (Cheng et al. 2020 , Liu et al. 2020). Tomato among the plants is considered the most vulnerable to environmental stress (Abewoy 2018). The different kinds of abiotic stresses such as drought, salt, heavy metals, and extreme temperature negatively affect every tomato life stage, causing about 70\% yield losses (Atkinson \&Urwin 2012, El-Sappah et al. 2019). The response to heavy metal stress involves a complicated signal transduction network that is activated by sensing the heavy metals and is characterized by the synthesis of stress-related proteins and signaling molecules, and finally, the transcriptional activation of specific metalresponsive genes to counteract the stress (El-Sappah et al. 2012). The typical consequence of most abiotic stresses, including heavy metal (BERTAMINI 2001), is the increased production of reactive oxygen species (ROS). These ROS [i.e. superoxide radical $\left(\mathrm{O}^{-}\right)$, hydrogen peroxide $\left(\mathrm{H}_{2} \mathrm{O}_{2}\right)$, hydroxyl $\left(\mathrm{OH}^{-}\right)$] are extremely toxic to plants (El-Sappah 2013, Maksymiec 2007). They caused damage to DNA, proteins, lipids and chlorophyll (Schutzendubel \&Polle 2002). However, plants are well equipped with an antioxidant system consisted of antioxidant enzymes (superoxide dismutase, peroxidase, catalase, glutathione reductase), low non-enzymatic molecule antioxidants (proline, tocopheroles, carotenoids, glutathione, ascorbic acid) to counter the oxidative stress to protect plants from oxidative injuries (Apel \&Hirt 2004). Accordingly, it is important to understand the mechanisms that help tomatoes withstand different stress conditions. Plants, including tomatoes, have advanced a range of mechanisms to acclimatize to altering environmental conditions (Becklin et al. 2016).

The molecular chaperones heat shock proteins (HSPs) are one sort of plant response to environmental stresses (El-Sappah et al. 2017, Sung et al. 2003). Several classes of HSP have been identified in plants with molecular weights ranging from 10 to 200KD, where the Hsp70 is one of them (El-Sappah et al. 2017). Hence, the induction of HSPs proteins is thought out as a critical protective, eco-physiologically adaptive, and genetically conserved response of organisms to environmental anxiety. Thus, they accomplish a key function in the hostility of stress by re-establishing normal protein conformation and cellular homeostasis (Rhee et al. 2009). Hsp70 families are highly conserved and encoded by a multi-gene family, which regulates the plant development as well as can be exist under different abiotic and biotic stresses (Liu et al. 2018, Song et al. 2019). In plants, Hsp70 gene families can be divided into four major subgroups with different cellular localization depending on their motifs at the C-terminus; plastids (PEGDVIDADFTDSK motif), mitochondria (PEAEYEEAKK) motif, cytosol (EEVD motif), and the endoplasmic reticulum (HDEL motif) (Luo et al. 2015).

The Hsp70 family has been identified in many plants such as arabidopsis 18 genes (Lin et al. 2001); potato 20 genes (Liu et al. 2018); soybean 61 genes (Zhang et al. 2015); pepper 21 genes (Guo et al. 2016), maize 22 genes (Jiang et al. 2020), and tobacco 61 genes (Song et al. 2019). Many heat shock proteins, especially the Hsp70, were induced by heavy metal stress (Khan et al. 2019). For instance, the Hsp70 sub-family, DnaK (Bip), was up-regulated in rice seedlings under the $\mathrm{Cd}$ and $\mathrm{Cu}$ contamination (Ahsan et al. 2007). A similar finding was in Populus trichocarpa, Glycine max, Arabidopsis thaliana (Lomaglio et al. 2015, Neumann et al. 1994). Furthermore, the cultured flax on heavy metal treated media induced many heavy metal-binding proteins, including Hsp70, while Hsp83 showed a down-regulation (Kosová et al. 2011).

Given the importance of resisting environmental hazards in tomatoes, and the whole tomato genome is now available (Wang et al. 2018), the identification and characterization of stress tolerance genes have become very urgent to tomato plant breeders and scientists. Therefore, the current study provides the first report genome-wide analysis of the $\mathrm{Hsp} 70$ gene family in tomato under the heavy metals stresses. We identified $23 \mathrm{Hsp} 70$ genes using bioinformatics methods. The phylogenic and the interaction within this gene family was also declared, as well as their expression profile of different tomato tissues under some stress was mentioned revealed to the previous RNA sequencing data. Furthermore, the expression of all $23 \mathrm{Hsp} 70$ genes under different heavy metals stresses was measured. Our data will be essential to understand the functional structure and the genomic organization of the $\mathrm{Hsp} 70 \mathrm{gene}$ members in the whole tomato genome that will be advantageous in further functional genomics characterization.

\section{Materials And Methods}

\subsection{The identification of Hsp70 genes in tomato}

The tomato sequences were obtained from the Solanaceae Genomics Network (https://solgenomics.net/) and then BioEide 7.0 software was used for the local database construction. The candidate tomato MTP genes were utilized used the HMM profile of the Hsp70 domain (Pfam: PF00012)

(http://www.sanger.ac.uk/Software/Pfam). The blast of putative Hsp70 protein sequences has been done compared to tomato whole genome, at the NCBI (http://blast.ncbi.nlm.nih.gov/blast.cgi), SPud DB tomato Solanaceae Genomics Network (https://solgenomics.net) and phytozome (https://phytozome.jgi.doe.gov/). 
The examination of all obtained protein sequences has been done at E-value $<10^{-5}$ for detecting the Hsp70 domain, using SMART (http://smart.emblheidelberg.de/)tools (Letunic et al. 2004). All genomic information about the selected $H s p 70$ gene family, such as chromosomal location and CDS, was obtained from the phytozome website database (https://phytozome.jgi.doe.gov/). The Hsp70 family's proteins were analyzed to obtain their characterization, such as the molecular weight, the number of atoms, the amino acid number, isoelectric point, and instability index using EXPASY PROTOPARAM (http://www.expasy.org/tools/protparam.html) (Gasteiger et al. 2003). Furthermore, the theoretical PI and molecular weight were obtained using ProtParam Tool (http://web.expasy.org/portparam).

\subsection{Phylogenetic analysis}

In addition to tomato, the potato and arabidopsis Hsp70 protein sequences have been downloaded from the Spud DB Potato Genomics Resources (http://solanaceae.plantbiology.msu.edu/) and the Arabidopsis Information Resources (TAIR) (http://arabidopsis.org). The CLUSTALX 2.0 software with default parameters has been used after that for Hsp70 proteins sequences multiple alignments. The previous alignment has been inserted into MEGA 6.0 software with a Neighbor-Joining method for the phylogenetic tree construction, and then, at last, the bootstrap analysis has been done at 1,000 iterations with a pair-wise gap deletion mode (Tamura et al. 2011).

\subsection{Chromosomal locations, synteny analysis, and protein-protein interactions}

The tomato gene database (https://phytozome.jgi.doe.gov/) supports us by the chromosomal position information of $H s p 70$ genes, which have been used to generate the genetics map by MapChart software. After that, two genes in the same species, located in the same clade of the phylogenetic tree, were defined as being coparalogs to identify whether tandem and segmental duplication events had occurred. On the other hand, the tomato gene database (https://phytozome.jgi.doe.gov/) has been further used with target genes for detecting the coordinates of the segmental duplications. The paralogs were regarded to be the results of tandem duplicated when two genes separated by five or fewer genes in a $100 \mathrm{~kb}$ region (Tang et al. 2008). Additionally, the coparalogs were considered segmental duplications if they were located on duplicated chromosomal blocks (Wei et al. 2007). Smith-Waterman algorithm (http://www.ebi.ac.uk/Tools/psa/) had been used for the calculation of the local alignments of two protein sequences. The synteny relationship with the chromosomal distribution for each SIHsp70 was introduced using circos (http://circos.ca/) (Krzywinski et al. 2009). Furthermore, for more knowledge about the cellular function of the Hsp70 protein family, the functional interactions between all expressed studied proteins are essential. The amino acid sequences of all the family members used for protein-protein interaction studies used the STRING database (https://string-db.org/).

\subsection{Gene structures and motif analyses}

The structure of all Hsp70 gene family members was analyzed to detect the intron/exon and their organization, and this has been done using both genomic and CDS sequences with the online tools of the Genes Structure Display Server program (GSDS, http://gsds.cbi.pku.edu.cn/index.php) (Hu et al. 2015). The conserved motif has been also detected for the gene family members using a Multiple EM for motif elicitation (MEME)

(http://meme.nbcr.net/meme3/meme.html) depends on the amino acid sequences (Bailey et al. 2006). The motif identification processes were adapted as a total maximum of 10 motifs in number and optimum motifs: 6 to 200 amino acid residues.

\subsection{Protein modeling, prediction and the gene ontology annotation (GO).}

The Phyre2 web has been used for protein modeling, prediction, and analysis of the SIHsp70 and this at intensive mode (sbg.bio.ic.ac.uk/phyre2/)(Kelley et al. 2015). Blast2GO v3.0.11 (https://www.blast2go.com) and OmicsBox sofware have been used with the identified Hsp70 protein sequences for GO annotation (Conesa \&Götz 2008).

\subsection{Gene expression analysis based on the RNA-seq data}

In our research, we checked the previous RNA-based data analyses for obtaining the real Hsp70 family genes expression during normal conditions. The expression data were downloaded from Tomato functional genomics databases (http://ted.bti.cornell.edu/pgsc_download.shtml) for some of the tomato organs such as leaves, roots, and flowers. Then the gene expression has been analyzed using the cufflinks (version: 2.2.1). Finally, after dividing absolute FPKM values by their mean and transformed the ratio by log2, the MeV 4.5 was used to cluster the expression data as a heat map (http://heatmapper.ca/) (Babicki et al. 2016, Saeed et al. 2006).

\subsection{Growth Conditions and Heavy Metal Treatments}

In this study, the tomato M82 line was cultivated during the Autom of 2020 at the experimental greenhouse of Yibin University (China). First, the seeds were washed with $10 \%$ hypochlorous acid and distilled water. The seeds have been germinated using water-saturated filter paper, and then transferred to fertilized pittmoss soil with germination conditions of 16 hours light $\left(27^{\circ} \mathrm{C}\right)$ and 8.0 dark $\left(18^{\circ} \mathrm{C}\right)$ with a relative humidity of $70 \%$. Thirty-day-old tomato were placed in $1 / 2$ Hoagland solutions ( $\mathrm{pH}$ 6.0) with different heavy metal treatments $0.1 \mathrm{mM} \mathrm{CdCl}_{2}, 0.1 \mathrm{mM} \mathrm{CoCl}_{2}, 0.5 \mathrm{mM} \mathrm{FeSO}_{4}, 1 \mathrm{mM} \mathrm{MnSO}_{4}$ and $0.5 \mathrm{mM} \mathrm{ZnSO}_{4}$ respectively, while normal 1/2 Hoagland solutions as the control (CK) (Desoky et al. 2019, Gao et al. 2020). Then, $24 \mathrm{~h}$ later, the leaves and roots of tube plantlets were collected and used as RNA extraction materials.

\section{8. qRT-PCR analysis}

The Trizol reagent (Invitrogen, USA) has been used to isolate the RNA from all plant samples (leaf and root), and the cDNA synthesis SuperMix Kit (Transgen, Beijing) used for the construction of the cDNA. The specific primers of all selected genes have been designed used Primer 5.0 (Table S1) with B-actin as a housekeeping gene. The real-time PCR was performed with the following reagents volumes: $10 \mu \mathrm{L}$ SYBR premix Taq $(2 \times)$ mixture, $1 \mu \mathrm{L}$ of $\mathrm{cDNA}, 0.5 \mu \mathrm{L}$ of each primer, and $8 \mu \mathrm{L}$ of $\mathrm{ddH}_{2} \mathrm{O}$ for a total volume of 20 . The cycles have been adapted as follows: $95^{\circ} \mathrm{C}$ for 10 minutes; 40 cycles at $95^{\circ} \mathrm{C}$ for 15 seconds, and $60^{\circ} \mathrm{C}$ for 60 seconds. The relative expression has been calculated based on the Livak equation of $2^{-\triangle \Delta C T}$ values for three replicates of every sample (Livak \&Schmittgen 2001). 


\section{Statistical analysis}

The Student's t-test has been used to calculate the data at a significance level of 0.05 in Excel software. Three biological replicates of expression analyses have been performed with \pm standard deviation (SD) at $p<0.05$. The significant variations between means were compared at $p<0.05$ (Duncan's Multiple Range Test). Furthermore, COSTAT computer software (CoHort Software version 6.303, Berkeley, CA, USA) was used for the statistical analysis.

\section{Results}

\subsection{The identification of $H s p 70$ genes in tomato}

A total set of 31 genes were identified at the beginning of the research, and after excluding the incompleted domain genes, we finally selected 23 candidate genes for further evaluation and study. The genes have been given new names from SIHsp70-1 to SIHsp70-23, as shown in Table 1. The whole set of tomato 12 chromosomes contributed to harboring the $H s p 70$ genes, except chromosome number five, which does not carry any of these genes. Furthermore, the molecular weight varied between all genes, ranging from 21273.47 to $98787.40 \mathrm{kDa}$. The total numbers of negative L-alpha-aspartyl and L-glutamic acid residues (Asp + Glu) were the dominant in all studied genes except SIHsp70-12. Moreover the total number of gene intron was varied between the studied family members except for the two genes $S / H s p 70-9$ and $S / H s p 70-13$, which have no intron. The content of every studied gene from their aminoacids were varied (ranging from 186 and 890 amino acids). Besides, all of the studied 23 genes have an acidic isoelectric point (PI), which indictor for $\mathrm{Hsp} 70$ genes rich content in acidic amino acids. 
Table 1

The characteristics of Hsp70 genes in tomato.

\begin{tabular}{|c|c|c|c|c|c|c|c|c|c|c|c|}
\hline $\begin{array}{l}\text { Hsp70 } \\
\text { gene }\end{array}$ & Sequance ID & Location & $(-)$ & $(+)$ & MW & aa & $\begin{array}{l}\text { Total } \\
\text { no. of } \\
\text { atoms }\end{array}$ & Instability & $\begin{array}{l}\text { Aliphatic } \\
\text { index }\end{array}$ & Intron & PI \\
\hline $\begin{array}{l}\text { SIHsp70- } \\
1\end{array}$ & Solyc01g106210 & SL2.50ch01:94157485..94161397 & 89 & 84 & 72969.84 & 681 & 10334 & 38.21 & 87.27 & 5 & 5.75 \\
\hline $\begin{array}{l}\text { SIHsp70- } \\
2\end{array}$ & Solyc06g076020 & SL2.50ch06:47192489..47195586 & 102 & 82 & 71008.48 & 648 & 9984 & 32.79 & 82.02 & 1 & 5.04 \\
\hline $\begin{array}{l}\text { SIHsp70- } \\
3\end{array}$ & Solyc03g082920 & SL2.50ch03:52794869..52798836 & 114 & 92 & 73457.21 & 667 & 10382 & 29.90 & 85.95 & 6 & 5.07 \\
\hline $\begin{array}{l}\text { SlHsp70- } \\
4\end{array}$ & Solyc10g086410 & SL2.50ch10:65236863..65240232 & 100 & 81 & 70779.21 & 644 & 9949 & 35.11 & 82.39 & 1 & 5.07 \\
\hline $\begin{array}{l}\text { SlHsp70- } \\
5\end{array}$ & Solyc01g106260 & SL2.50ch01:94215968..94220340 & 86 & 81 & 71876.57 & 670 & 10151 & 38.91 & 85.04 & 5 & 5.95 \\
\hline $\begin{array}{l}\text { SlHsp70- } \\
6\end{array}$ & Solyc07g043560 & SL2.50ch07:57457649..57465996 & 134 & 121 & 98787.40 & 890 & 13932 & 39.17 & 80.89 & 12 & 5.91 \\
\hline $\begin{array}{l}\text { SIHsp70- } \\
7\end{array}$ & Solyc02g080470 & SL2.50ch02:44673835..44685301 & 110 & 98 & 84108.25 & 753 & 11771 & 42.99 & 78.62 & 8 & 6.02 \\
\hline $\begin{array}{l}\text { SlHsp70- } \\
8\end{array}$ & Solyc06g052050 & SL2.50ch06:35713191..35716219 & 102 & 82 & 67513.37 & 619 & 9543 & 29.09 & 87.74 & 8 & 5.04 \\
\hline $\begin{array}{l}\text { SlHsp70- } \\
9\end{array}$ & Solyc03g117630 & SL2.50ch03:66724560..66726524 & 99 & 83 & 71849.40 & 654 & 10088 & 31.01 & 87.72 & 0 & 5.21 \\
\hline $\begin{array}{l}\text { SlHsp70- } \\
10\end{array}$ & Solyc01g099660 & SL2.50ch01:89839013..89842124 & 112 & 98 & 74641.87 & 669 & 10568 & 34.03 & 87.16 & 6 & 5.36 \\
\hline $\begin{array}{l}\text { SIHsp70- } \\
11\end{array}$ & Solyc07g005820 & SL2.50ch07:655717..659235 & 103 & 86 & 71953.43 & 654 & 10115 & 33.75 & 81.10 & 1 & 5.15 \\
\hline $\begin{array}{l}\text { SlHsp70- } \\
12\end{array}$ & Solyc03g117620 & SL2.50ch03:66722304..66723457 & 18 & 30 & 21273.47 & 186 & 2997 & 46.72 & 73.33 & 1 & 9.37 \\
\hline $\begin{array}{l}\text { SIHsp70- } \\
13\end{array}$ & Solyc09g075950 & SL2.50ch09:67581791..67583521 & 69 & 53 & 62723.17 & 576 & 8852 & 42.31 & 98.49 & 0 & 5.56 \\
\hline $\begin{array}{l}\text { SIHsp70- } \\
14\end{array}$ & Solyc11g020040 & SL2.50ch11:10015582..10019521 & 101 & 90 & 74493.21 & 692 & 10544 & 27.93 & 84.36 & 7 & 5.36 \\
\hline $\begin{array}{l}\text { SlHsp70- } \\
15\end{array}$ & Solyc11g066100 & SL2.50ch11:51773141..51775439 & 100 & 82 & 71458.91 & 654 & 10036 & 33.10 & 80.69 & 1 & 5.10 \\
\hline $\begin{array}{l}\text { SIHsp70- } \\
16\end{array}$ & Solyc04g011440 & SL2.50ch04:3894918..3898067 & 100 & 82 & 71389.83 & 651 & 10030 & 32.91 & 80.78 & 1 & 5.13 \\
\hline $\begin{array}{l}\text { SlHsp70- } \\
17\end{array}$ & Solyc12g043110 & SL2.50ch12:39110693..39115806 & 130 & 106 & 93882.82 & 852 & 13211 & 42.35 & 80.06 & 8 & 5.23 \\
\hline $\begin{array}{l}\text { SlHsp70- } \\
18\end{array}$ & Solyc12g043120 & SL2.50ch12:39096307..39100382 & 130 & 105 & 92996.38 & 846 & 13051 & 42.45 & 77.74 & 8 & 5.22 \\
\hline $\begin{array}{l}\text { SlHsp70- } \\
19\end{array}$ & Solyc08g082820 & SL2.50ch08:65489311..65493585 & 113 & 93 & 73200.96 & 666 & 10357 & 30.84 & 87.85 & 7 & 5.10 \\
\hline $\begin{array}{l}\text { SIHsp70- } \\
20\end{array}$ & Solyc08g079170 & SL2.50ch08:62804339..62810456 & 98 & 91 & 65165.56 & 579 & 9124 & 36.18 & 67.03 & 6 & 5.99 \\
\hline $\begin{array}{l}\text { SIHsp70- } \\
21\end{array}$ & Solyc01g103450 & SL2.50ch01:92060728..92065237 & 98 & 85 & 74896.54 & 703 & 10598 & 25.83 & 86.13 & 7 & 5.20 \\
\hline $\begin{array}{l}\text { SlHsp70- } \\
22\end{array}$ & Solyc11g066060 & SL2.50ch11:51740558..51743431 & 101 & 90 & 77141.77 & 698 & 10869 & 33.98 & 84.14 & 2 & 5.51 \\
\hline $\begin{array}{l}\text { SIHsp70- } \\
23\end{array}$ & Solyc09g010630 & SL2.50ch09:3965253..3968837 & 100 & 82 & 71224.69 & 649 & 10008 & 35.04 & 82.53 & 1 & 5.13 \\
\hline
\end{tabular}

\subsection{Phylogenetic analysis of $\mathrm{Hsp} 70$ gene families}

The phylogenetic tree was done using $19 \mathrm{Hsp} 70$ gene members from potato and 20 genes from arabidopsis besides the $23 \mathrm{Hsp} 70 \mathrm{~s}$ of tomato using their amino acid sequences with Neighbor-Joining methods at MEGA5.0 software and 100 replicants bootstrap value. The Hsp70 families, according to this tree, lie in four groups which were Group I, II, III, and IV (Fig. 1). Group, I contained large numbers of Hsp70 compared to other groups. It contained eight, nine, and six genes from tomato, potato, and arabidopsis, respectively, while group IV contained the second largest number of studied 23 genes of tomato, which were six genes. 


\subsection{Chromosomal locations and synteny analysis of $\mathrm{Hsp} 70$ gene family}

Further studies have been done on all members of the SIHsp70 gene family, which aimed to further identify their location on the tomato chromosomes and their effect on the gene duplication. As mentioned before, all sets of the $\mathrm{Hsp} 70$ genes are distributed on all tomato chromosomes except chromosome 5 , which does not harbor any genes of the studied $H s p 70$ family. Chromosomes 1,3, and 11 harbors the largest number of the $H s p 70$ gene family, where they carry ten whole of this family genes. Furthermore, for the evolution of novel functions and gene family expansion, we investigated gene duplication and divergence. Our results revealed segmental and tandem gene pair duplication from PGDD (Plant Genome Duplication Database) with circos for examining the duplications behavior on $\mathrm{Hsp} 70$ gene family members. One hundred twenty pairs of collinearity gene pairs with identity ranging from $50-100 \%$ were detected and these due to the exist of the two types of duplication, segment and tandem (Table S2). The segment duplication resulted in many homologies of Hsp70 genes between the chromosomes within the tomato genome, such as what occurs with the genes, SIHsp70-3, SIHsp70-10, and SIHsp70-13 (Fig. 2). On the other hand, there are five obvious tandem replicated gene clusters on chromosomes 1, 3, 8, 11, and 12.

\subsection{Phylogenetic analysis, gene structures, and motif analyses of the tomato Hsp70 gene family}

The Hsp70 gene members were divided into five subfamilies (A, B, C, D and E). Subfamily A was the largest among all the subfamilies with eight genes, followed by subfamily E with six genes, while subfamily D contains only one gene ( Fig. 3a). There was variation in exon-intron between different genes where they showed a high degree of similarity in the same subfamily that supports the close evolutionary relationships of tomato $H s p 70$ gene family members (Fig. 3c). Our analysis showed that all of the Hsp70 family members contained a greatly varied number from intron except in the two genes S/Hsp70-9 and SIHsp70-13 which have no intron. The largest intron is often indicated the complexity of gene structure. We analyzed all of the conserved motifs of $S / H s p 70$ using MEME depends on the amino acid sequences with ten motifs (Fig. 3b, and Table 2). All of the studied motifs contain 50 amino acids, except motif 4 , 6 , and 8 with 29 amino acids, motif 41,33, and 29, respectively. The largest number of common motifs were 1,3 and 8, which have been noticed within all the subfamilies except D, followed by the motifs 4,7 , and 6 , which disappear in only two of the studied Hsp70 genes. The number, type, or order of motifs pretty much was observed to similar within the same subfamily moreover between different families.

Table 2

Analysis of the 10 conserved motifs of SIHsp70 genes in tomato.

\begin{tabular}{|c|c|c|}
\hline Motif Logo & Best possible match & Width \\
\hline 1 & VKBAVVTVPAYFNDSQRQATKDAGVIAGLNVLRIINEPTAAAJAYGLDKK & 50 \\
\hline 2 & LLDVTPLSLGJETAGGVMTKLIPRNTTIPTKKEQVFSTYSDNQPGVLIQV & 50 \\
\hline 3 & EKNVLVFDLGGGTFDVSJLTIEEGIFEVKATAGDTHLGGEDFDNRLVNHF & 50 \\
\hline 4 & TRARFEELNMDLFRKCMEPVEKCLRDAKLDKSDIHEVVLVGGSTRIPKVQ & 41 \\
\hline 5 & EGERARTKDNNLLGKFELSGIPPAPRGVPQIEVCFDIDANGILNVSAEDK & 50 \\
\hline 6 & FNGKEPCKSINPDEAVAYGAAVQAAILSG & 33 \\
\hline 7 & ERLIGDAAKNQAAMNPENTVFDAKRLIGRRFSDP & 50 \\
\hline 8 & FKRKHKKDISGBPRALRRLRTACERAKRTLSSTAQTTIEIDSLYEGIDFY & 29 \\
\hline 9 & FKRKHKKDISGBPRALRRLRTACERAKRTLSSTAQTTIEIDSLYEGIDFY & 21 \\
\hline 10 & YKGEEKQFSPEEISAMVLTKMKEIAEAFL & 29 \\
\hline
\end{tabular}

\subsection{Protein modeling, prediction, protein-protein interactions and $\mathrm{GO}$ enrichment analysis}

Phyre 2 web portal for protein modeling (http://www.sbg.bio.ic.ac.uk/phyre2/html/page.cgi?id=index) has been processed using amino acid sequences of SIHsp70s (Fig. 4 and tables. S 3). The eleven predicted models of the SIHsp70s were based on c5tkyA_, c5e84B_, c3d2fC, c2khoA, c3c7nB_and c5obuA_ templates with $100 \%$. On other hand, the STRING investigation of protein-protein interactions showed the physical (direct) and the functional (indirect) associations (Fig. 5 and Table S4). The result showed different interactions within the studied proteins where the total number of nodes was 22 with an average of 8. 82. The STRING database analysis showed 97 edges with 14 expected one and showed 13 expected local network clusters, which were CL:4682, $4141,4138,4661,4411,4655,4020,4084,4021$, 4025, 3997, 4023 and 4339. Cluster 3997 was the biggest, containing 12 of the Hsp70 proteins (Table S5). Moreover, our protein analysis showed the appearance of two common domains within the checked members of the Hsp70 family, which were PF00012 (23 proteins) and PF06723 (19 proteins), (Table S6). Besides, our checked proteins family showed five pathways according to the Kyoto Encyclopedia of Genes and Genomes (KEGG), which were sly04141 (14 proteins), sly03060 ( 3 proteins), sly04144(10 proteins), sly03040 (10 proteins), and finally sly03018 ( two proteins) (Table S6). Similarly, sub-cellular localization, molecular function, and biological process were predicted by GO enrichment analysis (Fig. 6 and Table S5). In sub-cellular localization analysis, the predicted distribution scores of Hsp70 proteins were as following; $2 / 4 \%$ in vaculoar membranes, $3.5 / 8 \%$ in the chloroplast, and $9 / 42 \%$ in endoplasmic reticulum lumen. Noticeably, the $S / H s p 8$ gene was localized in 21 sub-cellular compartments out of all 28 , which underlined the significant role of $\mathrm{SIHsp} 8$ in metal stress resistance. Collective scores of Hsp70 protein molecules during biological processes were as following; response to heat and cadmium was $3 / 4 \%$, while cellular response to stress was $17 / 23 \%$ and cellular response to chemical stimulus was $15 / 23 \%$. More precisely, SIHsp8, SIHsp12, and SIHsp19 play a key role in response to different stresses. Molecular function analysis revealed significant roles of SIHsp12 and SIHsp23 in heavy metal processes.

\subsection{Gene expression analysis based on the RNA-seq data}


Previous RNA- RNA-seq data for the expression profile of the Hsp70 genes in different tomato tissues have been used for screening the expression level of these genes family in flower buds, opened flowers, $1 \mathrm{~cm}$ fruits, $2 \mathrm{~cm}$ fruits, $3 \mathrm{~cm}$ fruits, mature green fruits, breaker fruits, breaker +10 fruits, roots, and leaves (Fig. 7, and Table S8). Depending on previous RNA seq the highest expression levels were detected with SIHsp70-23 in all selected tissues except in breaker + 10 fruits, where the SIHsp70-15 recorded the highest level. Moreover, the lowest expression level was detected with the SIHsp70-10 and SIHsp70-11.

\section{7. qRT-PCR analysis of the SIHsp70 genes under different environmental stresses}

As shown in Fig. 8, different expression patterns of different SIHsp70s in tomato leaves and roots were observed under the five heavy metals stresses. The 23 Hsp70 genes showed differential vaulues with different types of heavy metals in root and leaf : In root, $\mathrm{Cd}^{2+}$ enhanced the expression of SIHsp70-1, SIHsp702, SIHsp70-3, SIHsp70-4, SIHsp70-5, SIHsp70-8, SIHsp70-10, SIHsp70-11, SIHsp70-12, SIHsp70-13, SIHsp70-19 and SIHsp70-23 but decreased the expression of SIHsp70-7, SIHsp70-17 and SIHsp70-20; $\mathrm{Co}^{2+}$ decreased the expression levels of SIHsp70-7, SIHsp70-18, SIHsp70-20 and SIHsp70-21 but increased the expression levels of SIHsp70-1, SIHsp70-3, SIHsp70-5, SIHsp70-8, SIHsp70-10, SIHsp70-11, SIHsp70-12, SIHsp70-13, SIHsp70-15, SIHsp70-19, and SIHsp70-23; the SIHsp70-3, SIHsp70-8, SIHsp70-17, SIHsp70-19 and SIHsp70-23, recorded the highest expression but the expression of other two genes SIHsp70-14 and SIHsp70-21 it decresd under $\mathrm{Mn}^{2+}$ contamination ; $\mathrm{Zn}^{2+}$ increased the expression of SIHsp70-3, SIHsp70-8, SIHsp70-10, SIHsp70-11, SIHsp70-12, SIHsp70-19 and SIHsp70-23 while it decresed the expression of SIHsp70-14, SIHsp70-18 and SIHsp70-21; Fe ${ }^{2+}$ increased the expression levels of SIHsp70-3, SIHsp70-8, SIHsp70-19 and SIHsp70-23 but decresed the expreseeion of SIHsp70-18, SIHsp70-20 and SIHsp70-21. In leaf, Cd ${ }^{2+}$ enhanced the expression of SIHsp70-1, SIHsp70-2, SIHsp70-3, SIHsp70-4, SIHsp70-5, SIHsp70-8, SIHsp70-10, SIHsp70-12, SIHsp70-13, SIHsp70-15 and SIHsp70-23 while decrese the expression of SIHsp70-7, SIHsp70-17 and SIHsp70-20, Co ${ }^{2+}$ increased the expression of SIHsp70-1, SIHsp70-3, SIHsp70-4, SIHsp70-5, SIHsp70-8, SIHsp70-10, SIHsp70-12, SIHsp70-13, SIHsp70-15 SIHsp70-19 and SIHsp70-23 but decreased the expression of SIHsp70-7, SIHsp70-14, SIHsp70-17 and SIHsp70-18; Fe ${ }^{2+}$ increased the expression of SIHsp70-17 and SIHsp70-23 but reduce the expression of SIHsp70-18, SIHsp70-20 and SIHsp70-21; Mn ${ }^{2+}$ decrease the expression of SIHsp7014 and SIHsp70-21 but enhanced the expreesion of SIHsp70-3, SIHsp70-8, SIHsp70-12 and SIHsp70-23; Zn ${ }^{2+}$, decrese the expression of SIHsp70-7, SIHsp70-14, SIHsp70-18 and SIHsp70-21 but enhanced the expression of SIHsp70-1, SIHsp70-3, SIHsp70-8, SIHsp70-10, SIHsp70-12 and SIHsp70-23.

\section{Discussion}

The Hsp70 considers one of the conserved protein family, which is essential for plants as it participates in multiple roles such as protection of other proteins during stress, assistance in protein translocation, and protein biogenesis (Ray et al. 2016). The Hsp70 has previously been studied in several plants, such as Arabidopsis (Lin et al. 2001), potato (Liu et al. 2018), pepper (Guo et al. 2016), and cotton (Rehman et al. 2020), while this is the first study of Hsp70 in tomato. Therefore, our study implements a genome-wide identification and expression analysis of the tomato $H s p 70$ gene family based on $H s p 70$ gene family selection, phylogeny, chromosomal locations, gene structure, motif sequences, protein-protein interactions, synteny analysis, and the expression profile based on previous RNA sequencing data or current environmental stresses treatments. The tomato $H s p 70$ gene family was 23 genes distributed in four groups based on the phylogenetic analysis of $\mathrm{Hsp} 70$ gene families in tomato, Arabidopsis, and potato (Fig. 1) as what mentioned in the other plants, such as the studies of Liu et al. (2018), who after phylogenetic analysis confirmed the exist of the four Hsp70 groups in potato. Furthermore, based on the phylogenetic tree and motif analysis of the tomato Hsp70 proteins, the studied family was consist of five subfamilies (Figs. 3), which are A, B, C, D, and E, where within the same sub-families, the most closely related and similar Hsp70 were found. This information related to gene structure from exons, introns, and motif sequences were identical to the previous studies in which a similar gene structure was found within the same subfamilies (Liu et al. 2018). For an instant, most of the gene members of subfamily A contain one intron. Howerver, most of subfamily $\mathrm{E}$ members contain eight introns, where as most of the subfamily $\mathrm{C}$ contains seven introns. These outcomes referred that during the structural evolution of tomato Hsp70, some intron gain and loss events may have occurred. Besides, the exons are lower than the introns in the gain/loss rate due to higher selection pressure in the exons sequences (Harrow et al. 2006). Besides, with all these observations, we have got to make sure that the placement divergences in intron number consider shared events that are more related to the evolution (Babicki et al. 2016, Jeffares et al. 2006, Rogozin et al. 2012).

Furthermore, the gene in which the introns exist and the host organism are two main determining factors of the intron evolutionary fate (Jeffares et al. 2006). In our study, to more knowledge about the gene annotation and the expansion mechanism of the Hsp70 gene family in tomatoes, we investigate the gene synteny and duplication analysis (Figs. 2 and Table S2). The exist of two or more genes on the same chromosome is often related to tandem duplication, while segmental duplication often occurs on different chromosomes (Schlueter et al. 2007). Our studies showed five tandem duplication pairs while there are more than 100 segmental duplications, such as the pairs SIHsp70-7/ SIHsp70-17, SIHsp70-7/ SIHsp70-18, and SIHsp70-10/ SIHsp70-15. In-plant gene families, the gene duplications events in its types followed by divergence, consider standard features and more related to secondary plant metabolism genes (Ober 2005). Hence, our results about the gene duplications confirmed their essential roles in the Hsp70 genes family expansion.

On the other hand, we used amino acid sequences of the SIHsp70s for predicting the protein 3D structure. Moreover some additional relevant information such as the structures of related proteins where its three-dimensional structure usually specifies the protein's function (Fig. 4 and tables S3) (Büyükköroğlu et al. 2018). The c3d2fC template (100\% confidence) has been used for modeling most of Hsp70 members except with (S/Hsp70-1 / SIHsp70-5), SIHsp70-12, SIHsp70-13 and SIHsp70-20 which used the c2khoA, c5gjjA, c5tkyA and C5nnrD templates respectively.

On the other hand, the protein-protein interaction considers an essential side of plant systems biology, which provides us more knowledge about the regulation of the plant developmental processes with plant and environmental interactions (Struk et al. 2019). Our protein-protein interactions analysis showed different interactions between the 23 proteins, which formed five pathways (Fig. 5).

On the other hand, gene ontology is a fundamental analysis to study each gene's different contributions across the living organisms (Consortium 2010). Moreover, gene ontology classes and concepts have been used to define the relationships and a gene function existing between these concepts (Purwantini et al. 2014). our gene ontology analysis showed the vital role of the tomato's SIHsp70 genes with heavy metals and other stresses (Fig. 6 and Table S7). 
Furthermore, the GO showed the Hsp70 genes' molecular function where more most of them participate in metal-related processes such as unfolded protein binding,protein folding chaperone \ion binding $\llbracket$ misfolded protein binding, and the oxidoreductase activity. HSP70 like DnaJ make a set of prominent cellular machines as aresult of the compination between the their chaperones with co-chaperones, to prevent the accumulation of newly synthesized proteins as aggregates and ensure the proper folding of protein during their transfer to the destination (Al-Whaibi 2011).

Thus, we investigated the expression profile of all members of the $\mathrm{Hsp} 70$ gene family from previously published RNA-sequencing data, which showed the expression of all gene members in all selected tomato tissues (Fig. 7 and Table S8). Worth evidence has been obtained about the essential roles of tomato $\mathrm{Hsp} 70$ genes after tissue expression evaluation. For instance, the exclusive increased expression as stage progress of the five genes $S / H s p 70-8, S / H s p 70-13$, SIHsp70-14, SIHsp70-20, and SIHsp70-23 in plant fruit, while 14 of the studied Hsp70 showed a significant increase in their expression at the open flower than the closed one indicating that they might participate in early fruit and flower development, respectively. Besides, the vital expected roles of $S / H s p 70-23$ in all plant tissues and developmental stages. However, only SIHsp70-10 and SIHsp70-11 were rarely expressed in all examined tissues from all SIHsp70 genes. The documented reduction in some gene expression is an essential factor for maintaining the gene duplicates and ancestral functions (Qian et al. 2010). Hence in our study, the reduction of SIHsp70-10 and SIHsp70-11 expression is expected to be vital for keeping their biological functions and maintain them from losing during the cell evaluation. Our finding agreed with Rehman et al. (2020), who investigated the differential expression analysis profiles of the Hsp70 family in cotton, which revealed their essential roles in plant development and tolerance. Generally, many Hsp70 genes have low expression while very few Hsp70 genes have high expression in developmental processes or specific organs (Chauhan et al. 2012, Neta-Sharir et al. 2005). There were 61, 32, 30, 18, 22, and 20 Hsp70 genes identified in Nicotiana tabacum, Oryza sativa, Gossypium raimondii, Arabidopsis thaliana, Zea mays and Solanum tuberosum, respectively (Jiang et al. 2020, Liu et al. 2018, Song et al. 2019). Most of the Hsp70 families in different plants exhibited different expression profiles under different environmental stresses (Zhang et al. 2015). The qRT-PCR has been processed for more authenticity after the transcriptome data analysis; however, the minor asymmetry between both analyses due to different growth conditions and tomato varieties, which finally affect the SIHsp70s expression. The transcription of Hsp70s in response to various heavy metals varied and complicated, although the gene expression response to different stresses is usually reflected in corresponding gene roles. In our study, the expression of Hsp70 members was different by a different type of heavy metals. For instant, the Cd ${ }^{2+}$ enhanced the expression of some genes such as SIHsp70-1, SIHsp70-2, SIHsp70-3, and SIHsp70-4, while it causes inhibition to others such as SIHsp70-7 and SIHsp70-14. The $\mathrm{Co}^{2+}$ enhanced the expression of some genes such as SIHsp70-15, SIHsp70-19, and SIHsp70-23 while it causes inhibition of others such as SIHsp70-7 and SIHsp70-14. $\mathrm{Zn}^{2+}$ increased some genes, such as SIHsp70-12 and SIHsp70-23, while it causes decreases in others. As the same as the Mn, which enhanced the expression of some genes such as SIHsp70-8 and SIHsp70-19 while it decreases the expression of SIHsp70-18 and SIHsp70-21. Finally, some

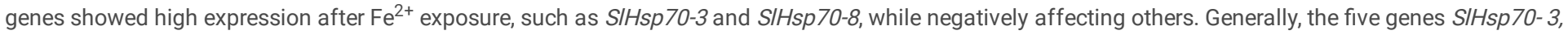
SIHsp70-8, SIHsp70-12, SIHsp70-19, and SIHsp70-23 showed the highest expression under heavy metal tratments. Our finding agreed with many of transcript analysis in many plant species in Populus trichocarpa (Lomaglio et al. 2015), Lycopersicon peruvianum L.(Neumann et al. 1994), Glycine max (Hossain et al. 2012), Arabidopsis thaliana (Sarry et al. 2006), Populus tremula (Kieffer et al. 2008), and Populus nigra (Lomaglio et al. 2015) which showed the high levels of their Hsp70 under the Cd stresses. Also, Spijkerman et al. (2007) studied the essential roles of HSPs 70A in Chlamydomonas acidophila under both Fe ${ }^{2+}$, and $\mathrm{Zn}^{2+}$ stresses. Another study by Basile et al. (2015) used the Hsp70 s as specific markers of heavy metals pollution in Lemna minor. Also, our finding agreed with Kosová et al. (2011), who recorded a high expression level of Hsp70 in flax after treated the cultured plant with the heavy metals while the Hsp83 showed down-regulation. Moreover, many other studies showed the essential roles of the Hsp70 with different heavy metal stresses in Arabidopsis (Lee \&Ahn 2013), Poplar (Augustine et al. 2015), and rice (Rodríguez-Celma et al. 2010). As more knowledge about the Hsp70 family is absent, our studies will be essential for investigating Hsp70s molecular roles in tomatoes under various heavy metals stresses.

\section{Conclusions}

Tomato plants, which are grown widely, are more sensitive to environmental stress than other plants due to their exposure to many environmental stresses such as heavy metals. Therefore, we performed bioinformatics and expression studies on the SIHsp 70 gene family, where we selected 23 genes in tomato as family members. The analysis of their organizations, distributions interactions, and structures supplied us with great evidence for the complicated evolutionary history of this studied family in tomato, as same as the protein-protein interaction and the 3D modeling showed. Moreover, the SIHsp70 gene family members showed many duplications distributed between tandem and segment duplications that are the major cause of the expansion of the genes. The RNA-seq data from previous studies besides the gene ontology indicated the multiple roles of these gene family members during the normal and abnormal environmental conditions. Finally, our expression analysis of $S / H s p 70$ genes at different tomato tissues showed different responses to heavy metals $\left(\mathrm{Cd}^{2+}, \mathrm{Co}^{2+}, \mathrm{Mn}^{2+}, \mathrm{Zn}^{2+}\right.$, and $\left.\mathrm{Fe}^{2+}\right)$. Our expression analysis revealed the significant roles of the Hsp70s, especially, SIHsp70-3, SIHsp70-8, SIHsp70-12, SIHsp 70-19, and SIHsp70-23, under heavy metals treatments. The comprehensive studies of this gene family will help facilitate additional research about the molecular functions and evolutionary history of the tomato $\mathrm{Hsp} 70$ gene family.

\section{Declarations}

\section{Acknowledgements:}

The authors are grateful and acknowledge Sichuan Province Government to provide such a well equipped platform to do research work, management of Yibin University for their support and providing us a pleasant environment of research, Chinese Government and Chinse Public in particular for their love of Science and Research.

\section{Author Contributions:}


Conceptualization, A.H.El-S (Ahmed H. El-Sappah) ; Formal analysis, A.H.El-S., S.A.E.S, M.A., and Y.Z; Methodology, A.H.El-S, H.E.El-S, V.Y., W.Y J.L and Q.H ; Writing-original draft, A.H.El-S.; writing-review and editing, A.H.El-S, and A.S.E ; Corresponding, Z.X, and J.L.

\section{Declaration of Competing Interest:}

The authors declared no conflict of financial interests or personal relationships that could have any influence on this research work.

\section{Funding}

Not applicable

\section{Data availability}

Not applicable

\section{Conflict of interest}

The authors declare that they have no conflict of interest.

\section{Consent to publish}

Not applicable

\section{Consent to participate}

Not applicable

\section{References}

1. Abewoy D (2018): Review on impacts of climate change on vegetable production and its management practices. Advances in Crop Science and Technology 6, 1-7

2. Ahsan N, Lee S-H, Lee D-G, Lee H, Lee SW, Bahk JD, Lee B-H (2007): Physiological and protein profiles alternation of germinating rice seedlings exposed to acute cadmium toxicity. Comptes rendus biologies 330, 735-746

3. Al-Whaibi MH (2011): Plant heat-shock proteins: a mini review. Journal of King Saud University-Science 23, 139-150

4. Apel K, Hirt H (2004): Reactive oxygen species: metabolism, oxidative stress, and signal transduction. Annu. Rev. Plant Biol. 55, 373-399

5. Atkinson NJ, Urwin PE (2012): The interaction of plant biotic and abiotic stresses: from genes to the field. Journal of experimental botany $63,3523-3543$

6. Augustine SM, Cherian AV, Syamaladevi DP, Subramonian N (2015): Erianthus arundinaceus HSP70 (EaHSP70) acts as a key regulator in the formation of anisotropic interdigitation in sugarcane (Saccharum spp. hybrid) in response to drought stress. Plant and Cell Physiology 56, 2368-2380

7. Babicki S, Arndt D, Marcu A, Liang Y, Grant JR, Maciejewski A, Wishart DS (2016): Heatmapper: web-enabled heat mapping for all. Nucleic acids research 44, W147-W153

8. Bailey TL, Williams N, Misleh C, Li WW (2006): MEME: discovering and analyzing DNA and protein sequence motifs. Nucleic acids research 34, W369W373

9. Basile A, Sorbo S, Cardi M, Lentini M, Castiglia D, Cianciullo P, Conte B, Loppi S, Esposito S (2015): Effects of heavy metals on ultrastructure and Hsp70 induction in Lemna minor L. exposed to water along the Sarno River, Italy. Ecotoxicology and environmental safety 114, 93-101

10. Becklin KM, Anderson JT, Gerhart LM, Wadgymar SM, Wessinger CA, Ward JK (2016): Examining plant physiological responses to climate change through an evolutionary lens. Plant physiology 172, 635-649

11. BERTAMINI M (2001): Triacontanol can protect Erythrina variegata from cadmium toxicity. Journal of plant physiology

12. Büyükköroğlu G, Dora DD, Özdemir F, Hızel C (2018): Chapter 15 Techniques for Protein Analysis, Omics Technologies and Bio-Engineering, pp. $317-351$

13. Chauhan H, Khurana N, Nijhavan A, Khurana JP, Khurana P (2012): The wheat chloroplastic small heat shock protein (sHSP26) is involved in seed maturation and germination and imparts tolerance to heat stress. Plant, cell \& environment 35, 1912-1931

14. Cheng G, Chang P, Shen Y, Wu L, El-Sappah AH, Zhang F, Liang Y (2020): Comparing the Flavor Characteristics of 71 Tomato (Solanum lycopersicum) Accessions in Central Shaanxi. Frontiers in plant science 11

15. Conesa A, Götz S (2008): Blast2GO: A Comprehensive Suite for Functional Analysis in Plant Genomics. International Journal of Plant Genomics 2008, 619832

16. Consortium GO (2010): The Gene Ontology in 2010: extensions and refinements. Nucleic acids research 38, D331-D335 
17. Desoky E, Elrys AS, Rady MM (2019): Licorice root extract boosts Capsicum annuum L. production and reduces fruit contamination on a heavy metalscontaminated saline soil. International Letters of Natural Sciences 73

18. El-Sappah A, Shawky A, Sayed-Ahmad M, Youssef M (2012): Nile tilapia as bio indicator to estimate the contamination of water using SDS-PAGE and RAPDPCR techniques. Egyptian Journal of Genetics and Cytology 41, 209-227

19. El-Sappah AH, Shawky A, Sayed-Ahmad MS, Youssef M (2017): Estimation of heat shock protein 70 (hsp 70) gene expression in nile tilapia (Oreochromis niloticus) using quantitative Real-Time PCR. Zagazig Journal of Agricultural Research 44, 1003-1015

20. El-Sappah AH, MM I, H El-awady H, Yan S, Qi S, Liu J, Cheng G-t, Liang Y (2019): Tomato natural resistance genes in controlling the root-knot nematode. Genes 10, 925

21. El-Sappah AHAM 2013: Utilization of Micronucleus Test and Genetic Markers for Determining The Pollution of Nile Tilapia (Oreochromis niloticus), Zagazig University

22. Gao Y, Yang F, Liu J, Xie W, Zhang L, Chen Z, Peng Z, Ou Y, Yao Y (2020): Genome-wide identification of metal tolerance protein genes in Populus trichocarpa and their roles in response to various heavy metal stresses. International journal of molecular sciences 21,1680

23. Gasteiger E, Gattiker A, Hoogland C, Ivanyi I, Appel RD, Bairoch A (2003): ExPASy: the proteomics server for in-depth protein knowledge and analysis. Nucleic acids research 31, 3784-3788

24. Guo M, Liu J-H, Ma X, Zhai Y-F, Gong Z-H, Lu M-H (2016): Genome-wide analysis of the Hsp70 family genes in pepper (Capsicum annuum L.) and functional identification of CaHsp70-2 involvement in heat stress. Plant Science 252, 246-256

25. Harrow J, Denoeud F, Frankish A, Reymond A, Chen C-K, Chrast J, Lagarde J, Gilbert JG, Storey R, Swarbreck D (2006): GENCODE: producing a reference annotation for ENCODE. Genome biology 7, 1-9

26. Hossain Z, Makino T, Komatsu S (2012): Proteomic study of $\beta$-aminobutyric acid-mediated cadmium stress alleviation in soybean. Journal of proteomics $75,4151-4164$

27. Hu B, Jin J, Guo A-Y, Zhang H, Luo J, Gao G (2015): GSDS 2.0: an upgraded gene feature visualization server. Bioinformatics 31, $1296-1297$

28. Jeffares DC, Mourier T, Penny D (2006): The origin of introns. Trends in Genetics 1, 16-22

29. Jiang L, Hu W, Qian Y, Ren Q, Zhang J (2020): Genome-wide identification, classification and expression analysis of the Hsf and Hsp70 gene families in maize. Gene 770, 145348

30. Kelley LA, Mezulis S, Yates CM, Wass MN, Sternberg MJE (2015): The Phyre2 web portal for protein modeling, prediction and analysis. Nature Protocols $10,845-858$

31. Khan A, Ali M, Khattak AM, Gai W-X, Zhang H-X, Wei A-M, Gong Z-H (2019): Heat shock proteins: dynamic biomolecules to counter plant biotic and abiotic stresses. International journal of molecular sciences 20, 5321

32. Kieffer P, Dommes J, Hoffmann L, Hausman JF, Renaut J (2008): Quantitative changes in protein expression of cadmium-exposed poplar plants. Proteomics 8, 2514-2530

33. Kosová K, Vítámvás P, Prášil IT, Renaut J (2011): Plant proteome changes under abiotic stress-contribution of proteomics studies to understanding plant stress response. Journal of proteomics $74,1301-1322$

34. Krzywinski M, Schein J, Birol I, Connors J, Gascoyne R, Horsman D, Jones SJ, Marra MA (2009): Circos: an information aesthetic for comparative genomics. Genome research 19, 1639-1645

35. Lee J, Ahn Y-J (2013): Heterologous expression of a carrot small heat shock protein increased Escherichia coli viability under lead and arsenic stresses. HortScience 48, 1323-1326

36. Letunic I, Copley RR, Schmidt S, Ciccarelli FD, Doerks T, Schultz J, Ponting CP, Bork P (2004): SMART 4.0: towards genomic data integration. Nucleic acids research 32, D142-D144

37. Lin B-L, Wang J-S, Liu H-C, Chen R-W, Meyer Y, Barakat A, Delseny M (2001): Genomic analysis of the Hsp70 superfamily in Arabidopsis thaliana. Cell stress \& chaperones 6, 201

38. Liu J, Pang X, Cheng Y, Yin Y, Zhang Q, Su W, Hu B, Guo Q, Ha S, Zhang J (2018): The Hsp70 gene family in Solanum tuberosum: genome-wide identification, phylogeny, and expression patterns. Scientific reports 8, 1-11

39. Liu J, Shi M, Wang J, Zhang B, Li Y, Wang J, El-Sappah A, Liang Y (2020): Comparative Transcriptomic Analysis of the Development of Sepal Morphology in Tomato (Solanum Lycopersicum L.). International journal of molecular sciences 21, 5914

40. Livak KJ, Schmittgen TD (2001): Analysis of relative gene expression data using real-time quantitative PCR and the 2- $\triangle \triangle C T$ method. methods 25 , $402-$ 408

41. Lomaglio T, Rocco M, Trupiano D, De Zio E, Grosso A, Marra M, Delfine S, Chiatante D, Morabito D, Scippa GS (2015): Effect of short-term cadmium stress on Populus nigra L. detached leaves. Journal of Plant Physiology 182, 40-48

42. Luo S, Ahola V, Shu C, Xu C, Wang R (2015): Heat shock protein 70 gene family in the Glanville fritillary butterfly and their response to thermal stress. Gene 556, 132-141

43. Maksymiec W (2007): Signaling responses in plants to heavy metal stress. Acta Physiologiae Plantarum 29, 177

44. Neta-Sharir I, Isaacson T, Lurie S, Weiss D (2005): Dual role for tomato heat shock protein 21: protecting photosystem II from oxidative stress and promoting color changes during fruit maturation. The Plant Cell 17, 1829-1838

45. Neumann D, Lichtenberger O, Günther D, Tschiersch K, Nover L (1994): Heat-shock proteins induce heavy-metal tolerance in higher plants. Planta 194, 360-367

Page $10 / 19$ 
46. Ober D (2005): Seeing double: gene duplication and diversification in plant secondary metabolism. Trends in plant science 10, 444-449

47. Purwantini E, Torto-Alalibo T, Lomax J, Setubal JC, Tyler BM, Mukhopadhyay B (2014): Genetic resources for methane production from biomass described with the Gene Ontology. Frontiers in microbiology 5, 634

48. Qian W, Liao B-Y, Chang AY-F, Zhang J (2010): Maintenance of duplicate genes and their functional redundancy by reduced expression. Trends in Genetics 26, 425-430

49. Ray D, Ghosh A, Mustafi SB, Raha S (2016): Plant stress response: Hsp70 in the spotlight, Heat Shock Proteins and Plants. Springer, pp. $123-147$

50. Rehman A, Atif RM, Qyyum A, Du X, Hinze L, Azhar MT (2020): Genome-wide identification and characterization of HSP70 gene family in four species of cotton. Genomics

51. Rhee J-S, Raisuddin S, Lee K-W, Seo JS, Ki J-S, Kim I-C, Park HG, Lee J-S (2009): Heat shock protein (Hsp) gene responses of the intertidal copepod Tigriopus japonicus to environmental toxicants. Comparative Biochemistry and Physiology Part C: Toxicology \& Pharmacology 149, 104-112

52. Rodríguez-Celma J, Rellán-Álvarez R, Abadía A, Abadía J, López-Millán A-F (2010): Changes induced by two levels of cadmium toxicity in the 2-DE protein profile of tomato roots. Journal of Proteomics 73, 1694-1706

53. Rogozin IB, Carmel L, Csuros M, Koonin EV (2012): Origin and evolution of spliceosomal introns. Biology direct 7, 1-28

54. Saeed Al, Bhagabati NK, Braisted JC, Liang W, Sharov V, Howe EA, Li J, Thiagarajan M, White JA, Quackenbush J (2006): [9] TM4 microarray software suite. Methods in enzymology 411, 134-193

55. Sarry JE, Kuhn L, Ducruix C, Lafaye A, Junot C, Hugouvieux V, Jourdain A, Bastien O, Fievet JB, Vailhen D (2006): The early responses of Arabidopsis thaliana cells to cadmium exposure explored by protein and metabolite profiling analyses. Proteomics 6, 2180-2198

56. Schlueter JA, Lin J-Y, Schlueter SD, Vasylenko-Sanders IF, Deshpande S, Yi J, O'bleness M, Roe BA, Nelson RT, Scheffler BE (2007): Gene duplication and paleopolyploidy in soybean and the implications for whole genome sequencing. BMC genomics 8, 1-16

57. Schutzendubel A, Polle A (2002): Plant responses to abiotic stresses: heavy metal-induced oxidative stress and protection by mycorrhization. Journal of experimental botany $53,1351-1365$

58. Song Z, Pan F, Lou X, Wang D, Yang C, Zhang B, Zhang H (2019): Genome-wide identification and characterization of Hsp70 gene family in Nicotiana tabacum. Molecular biology reports 46, 1941-1954

59. Spijkerman E, Barua D, Gerloff-Elias A, Kern J, Gaedke U, Heckathorn SA (2007): Stress responses and metal tolerance of Chlamydomonas acidophila in metal-enriched lake water and artificial medium. Extremophiles 11, 551-562

60. Struk S, Jacobs A, Sánchez Martín-Fontecha E, Gevaert K, Cubas P, Goormachtig S (2019): Exploring the protein-protein interaction landscape in plants. Plant, cell \& environment 42, 387-409

61. Sung D-Y, Kaplan F, Lee K-J, Guy CL (2003): Acquired tolerance to temperature extremes. Trends in plant science 8, 179-187

62. Tamura K, Peterson D, Peterson N, Stecher G, Nei M, Kumar S (2011): MEGA5: molecular evolutionary genetics analysis using maximum likelihood, evolutionary distance, and maximum parsimony methods. Molecular biology and evolution 28, 2731-2739

63. Tang H, Bowers JE, Wang X, Ming R, Alam M, Paterson AH (2008): Synteny and collinearity in plant genomes. Science 320, 486-488

64. Wang Y, Jiang J, Zhao L, Zhou R, Yu W, Zhao T (2018): Application of whole genome resequencing in mapping of a tomato yellow leaf curl virus resistance gene. Scientific reports $8,1-11$

65. Wei F, Coe E, Nelson W, Bharti AK, Engler F, Butler E, Kim H, Goicoechea JL, Chen M, Lee S (2007): Physical and genetic structure of the maize genome reflects its complex evolutionary history. PLoS Genet 3, e123

66. Zhang L, Zhao H-K, Dong Q-I, Zhang Y-Y, Wang Y-M, Li H-Y, Xing G-J, Li Q-Y, Dong Y-S (2015): Genome-wide analysis and expression profiling under heat and drought treatments of HSP70 gene family in soybean (Glycine max L.). Frontiers in plant science 6, 773

\section{Figures}




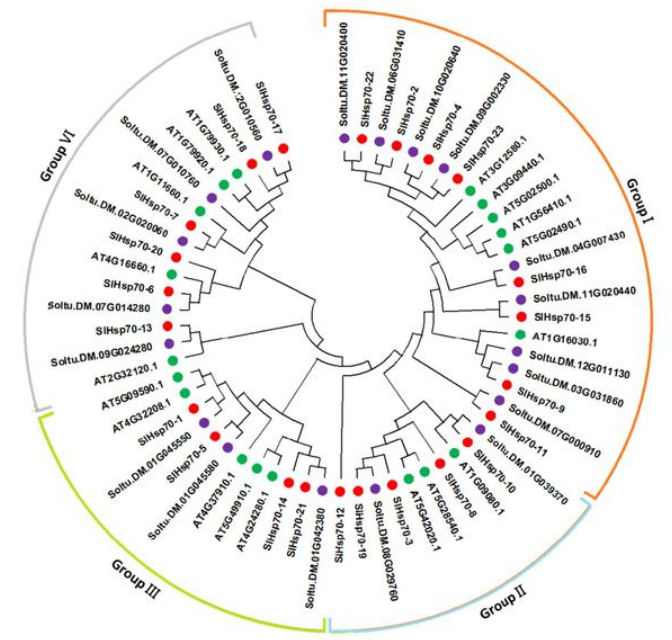

Figure 1

\section{Figure 1}

Phylogenetic tree of the $61 \mathrm{Hsp} 70$ proteins: 23 Tomato (marked by red circle) ,19 Arabidopsis ( green circle) and potato 19 (move circle). ClustalX1.83 was used for protein alignments and the phylogenetic tree's construction Neighbor-Joining (NJ) level with MEGA5.0 software at 1,000 replications bootstrap. 


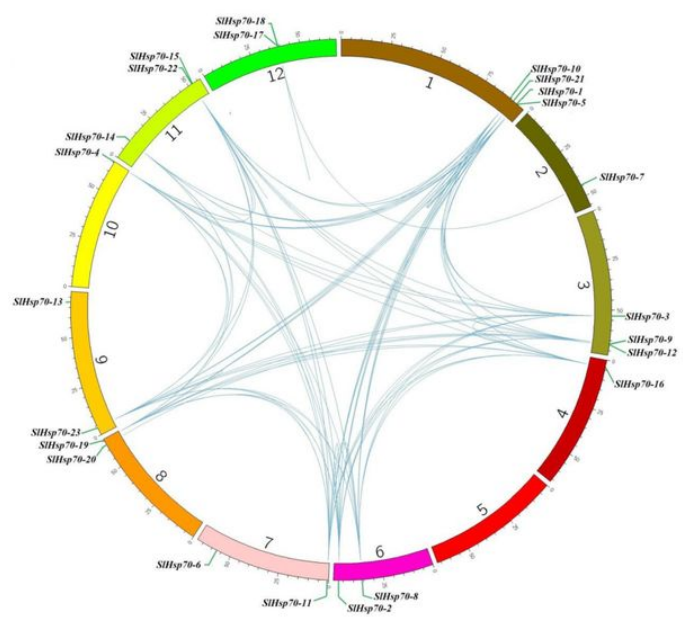

Figure 2

\section{Figure 2}

Genome-wide synteny analysis for Hsp70 gene family at 12 tomato chromosomes. The blue lines represented the syntenic orthologs and paralogs and showed the segment duplication while the he tandem duplicated genes were referred by red boxes. 


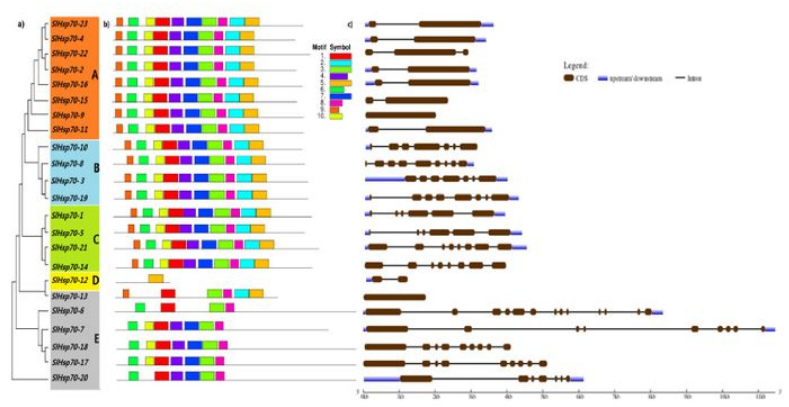

Figure 3

\section{Figure 3}

Phylogenetic relationship, gene structure and conserved motif analysis of SIHsp70 genes; a) The neighbor-joining phylogenetic tree was constructed with MEGA7 using SIHsp70 amino acid sequences with 1000 times replicate. b) The motif composition of SIHsp70 proteins using ten conserved motifs is represented by the unique colour mentioned in the box on the top lift. c) Exon-intron structure of the Hsp70 tomato proteins where dark green boxes presented the exons, and the black lines represent the introns. The blue boxes represented the untranslated regions (UTRs), with size scales detailed at the bottom. 

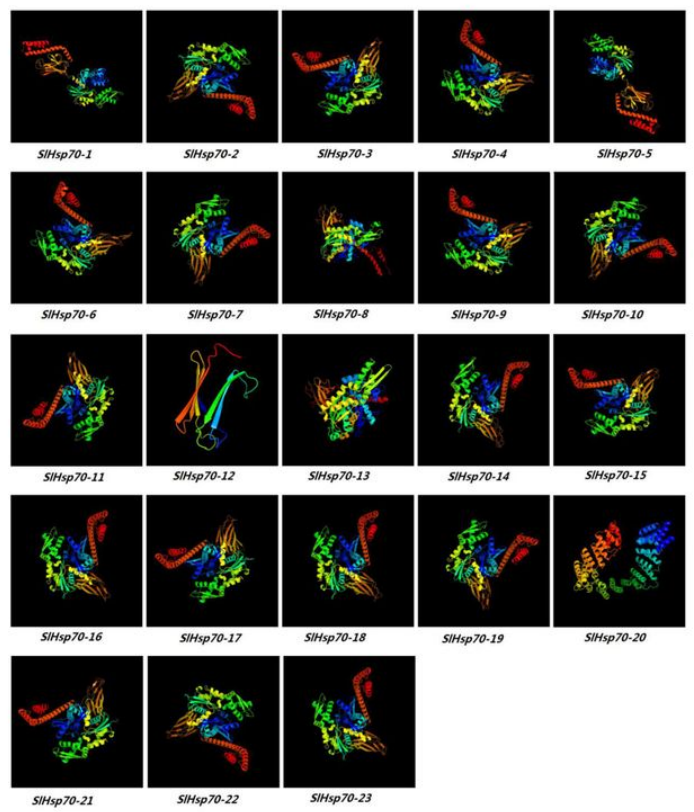

Figure 4

\section{Figure 4}

Predicted 3D models of tomato SIHsp70 proteins. Models have been generated by using the Phyre 2 server in intensive mode. Models were visualized by rainbow colour from $\mathrm{N}$ to $\mathrm{C}$ terminus and organized in SIHsp70-1, SIHsp70-2,...to SIHsp70-23, 


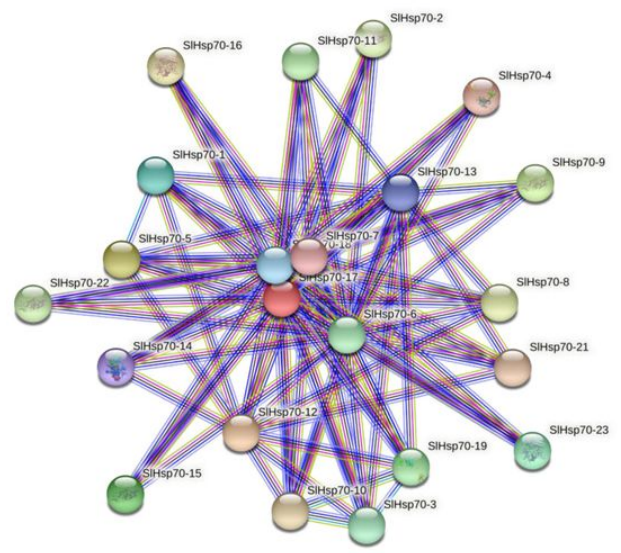

Figure 5

\section{Figure 5}

protein-protein interaction among the Hsp70 family members in tomato. 


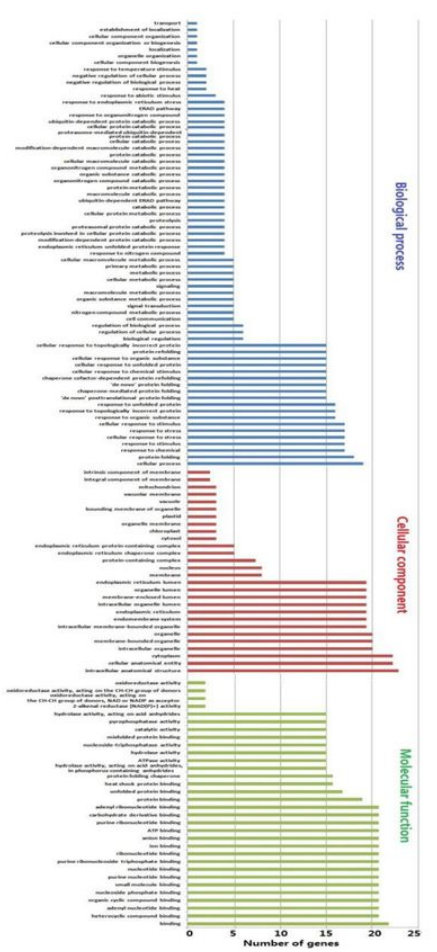

Figure 6

\section{Figure 6}

Gene Ontology analysis of tomato Hsp70 genes. Gene ontology showed the distribution of every SIHsp70 genes in the plant, where a red colour column mentioned the cellular component. In contrast, the biological processes in which the Hsp70 family participate were mentioned by the blue colour column, and the molecular function was mentioned by green colour. 


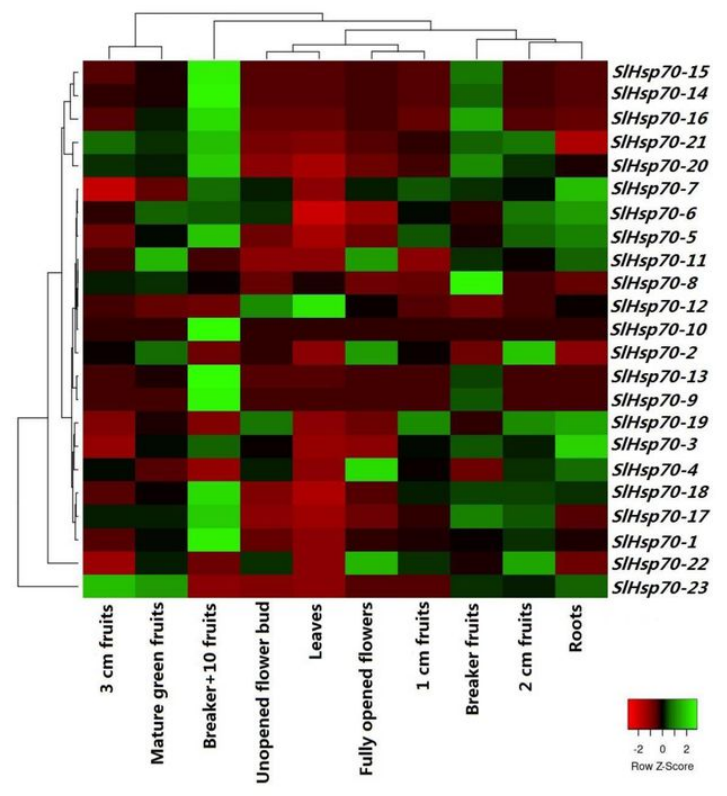

Figure 7

\section{Figure 7}

The heat map of the $23 \mathrm{SIHsp70} \mathrm{genes} \mathrm{expression} \mathrm{profiles} \mathrm{in} \mathrm{different} \mathrm{tomato} \mathrm{tissues} \mathrm{based} \mathrm{on} \mathrm{the} \mathrm{RNA-seq} \mathrm{(http://ted.bti.cornell.edu/).}$ 


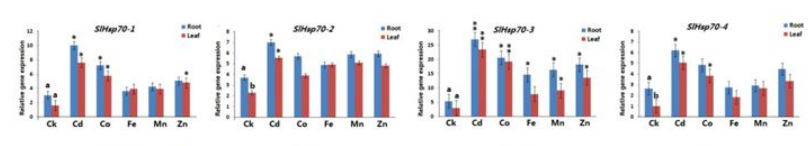

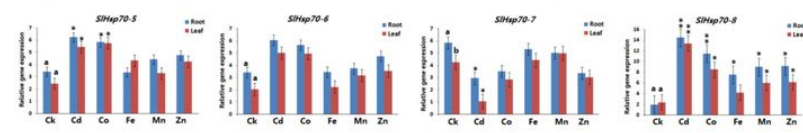

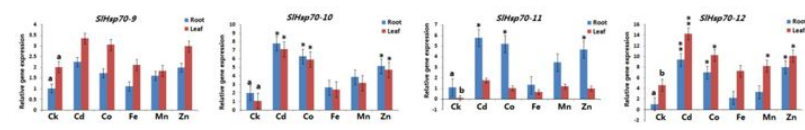

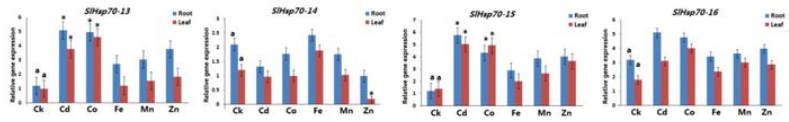

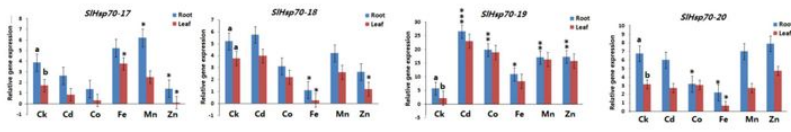

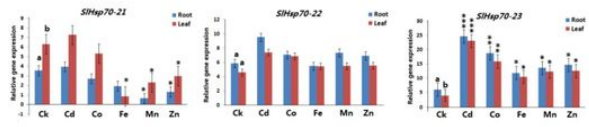

Figure 8

\section{Figure 8}

The qRT-PCR expression of the tomato SIHsp70 genes from root and leaf samples under various metal ion stresses. The reactions were normalized using the $\beta$-actin reference gene. The standard deviations have been represented by the error bars from three independent technical replicates. The mean expression levels of three replicates were analyzed with the five heavy metals treatments ( $\mathrm{Cd} 2+, \mathrm{Co} 2+, \mathrm{Fe} 2+, \mathrm{Mn} 2+$ and $\mathrm{Zn} 2+)$ using t-tests ( $\mathrm{p}<0.05)$ while the CK represent control samples. Asterisks indicate significant differences between the treatment samples and the corresponding control samples in roots and leaves. ( $n=9, p<0.05$, Student's t-test).

\section{Supplementary Files}

This is a list of supplementary files associated with this preprint. Click to download.

- Geneontology.xIsx

- S5.clusters.xIsx

- TableS1.primersforHsp70familyexpression.docx

- Tables2SI.MTP.synteny.xIsx

- Tables6.domain.xIsx

- previousRNAseq.xIsx

- renamedc7acf.xlsx

- stringinteractions.xlsx 